Check for updates

Cite this: RSC Adv., 2019, 9, 18619

\title{
Thermal- and salt-activated shape memory hydrogels based on a gelatin/polyacrylamide double network $\dagger$
}

\author{
Fang Chen, Kaixiang Yang, Dinglei Zhao and Haiyang Yang (DD* \\ Shape memory hydrogels have been extensively studied in the past decades owing to their exceptionally \\ promising potential in a wide range of applications. Here, we present a gelatin/polyacrylamide double \\ network hydrogel with thermal- and salt-activated shape memory effect. The thermally activated \\ behavior is attributed to the reversible triple helix transformation of gelatin, and the salt-activated \\ performance can be ascribed to the formation of hydrophobic interaction domains under the \\ Hofmeister effect. The hydrogel can memorize a temporary shape successfully through soaking with \\ $\left(\mathrm{NH}_{4}\right)_{2} \mathrm{SO}_{4}$ solution or decreasing temperature, and recovers its permanent shape by extracting ions with \\ deionized water or increasing temperature. In particular, the hydrogel exhibits excellent shape fixity and \\ recovery ratio. The presented strategy may enrich the construction as well as application of biopolymer \\ based shape memory hydrogels.
}

Received 15th April 2019

Accepted 24th May 2019

DOI: $10.1039 / \mathrm{c} 9 \mathrm{ra0} 2842 \mathrm{k}$

rsc.li/rsc-advances

adamantyl-terminated 8-arm PEG and a star shaped PNIPAAm with a cyclodextrin core. In addition, salt-activated shape memory performance was also paid much attention. ${ }^{21}$ Chen and co-workers ${ }^{22}$ reported the polyacrylamidechitosan (PAAm-CS) based shape memory hydrogel which was fabricated based on reversible physical interactions, microcrystal and chain-entanglement interactions. Here, chitosan can not only form a kind of chain-entangled structure in $\mathrm{NaCl}$ solution but also display a kind of microcrystalline structure in $\mathrm{NaOH}$ solution. Besides, there are also some SMHs triggered by orthogonal stimuli. ${ }^{23}$ We have previously reported the unusual thermal- and light-activated shape memory effects in an agarose/poly (acrylamide-coacrylic acid) interpenetrating network hydrogel. ${ }^{24}$ The thermal-activated shape memory behavior is attributed to the reversible coil-helix transition of agarose macromolecules in response to temperature while the light-activated SME is a result of the photoreduction of $\mathrm{Fe}(\mathrm{III})$ to $\mathrm{Fe}(\mathrm{II})$ in the presence of reductive citric acid.

In comparison to the synthetic polymer, biopolymers are more attractive for fabricating biocompatible SMHs. ${ }^{25}$ In particular, natural gelatin, as a representative derivative of collagen, possesses distinctive biocompatibility. ${ }^{26}$ With temperature increasing, gelatin exhibits reversible triple helices transformation and decrease of mechanical properties. $^{27}$ Recently, Wang groups ${ }^{28}$ reported that gelatin hydrogels which treated with $\left(\mathrm{NH}_{4}\right)_{2} \mathrm{SO}_{4}$ solution had more excellent ductility and strength due to Hofmeister effect. Hofmeister effect was the interactions between the ions and the polymer chains, which can be easily found in the process

CAS Key Laboratory of Soft Matter Chemistry, School of Chemistry and Materials Science, University of Science and Technology of China, Hefei 230026, China. E-mail: yhy@ustc.edu.cn; Tel: +86-551-63607549

$\dagger$ Electronic supplementary information (ESI) available. See DOI: $10.1039 / \mathrm{c} 9 \mathrm{ra} 02842 \mathrm{k}$ 
of folding and precipitation of proteins. ${ }^{29}$ However, SMHs based on Hofmeister effect have been rarely reported.

In the present work, we presented a thermal- and saltactivated shape memory hydrogel featuring for the gelatin/ polyacrylamide double network (gelatin/PAM DN). As depicted in Scheme 1, the gelatin/PAM hydrogel was prepared by free radical polymerization of AM in the presence of gelatin. The reversible configuration of the gelatin under different temperature endowed hydrogel with temperature responsive ability. Salt-activated performance was ascribed to the formation of reversible chain bundle and hydrophobic domains under Hofmeister effect. Thus, we here presented a new concept to fabricate biopolymerbased SMHs, which contributed to the development of smart soft materials.

\section{Experimental}

\section{Materials}

Gelatin (Porcine skin, Type A, 300 Bloom) was purchased from Sigma-Aldrich Life Science \& Technology Co., Ltd. Acrylamide (AM), ammonium sulfate, sodium carbonate, potassium carbonate, dipotassium hydrogenphosphate were obtained from Sinopharm Chemical Reagent Co., Ltd. $N, N^{\prime}$-Methylenebisacrylamide (MBA) and 2-hydroxy-4'-(2-hydroxyethoxy)-2methylpropiophenone (IRGACURE 2959) were purchased from Energy Chemical. All materials were analytical reagents and

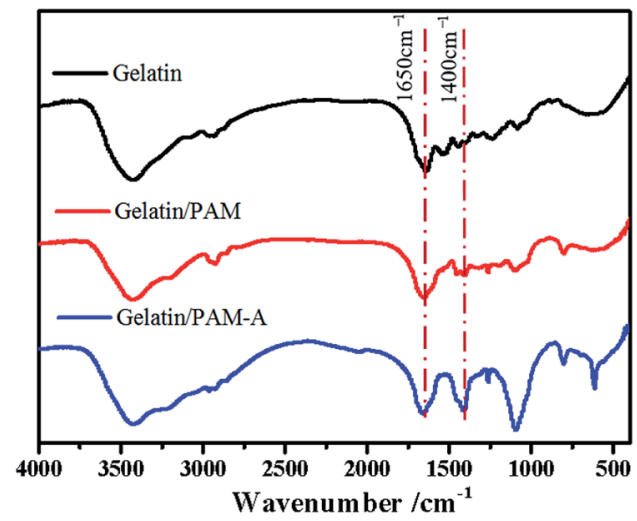

Fig. 1 The FTIR spectra of the gelatin, gelatin/PAM hydrogels, and gelatin/PAM-A hydrogels.

used as received without further purification. Deionized water was used in all the experiments.

\section{Preparation of gelatin/PAM double network hydrogels}

The synthesis of hydrogels was briefly described here. First, the suspension of gelatin in deionized water was heated at $50{ }^{\circ} \mathrm{C}$ for 30 min to obtain a transparent solution. Then, AM, MBA (chemical cross-linker) and IRGACURE 2959 as a photoinitiator were dissolved in the above solution. After removing all air bubbles by ultrasonication, the homogenous mixture was poured into molds

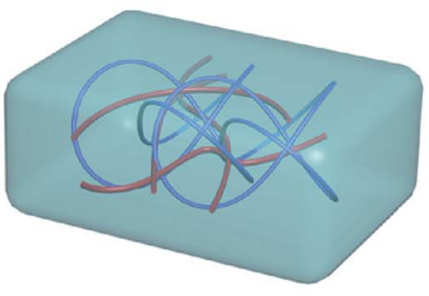

$\circ \mathbf{A M}$
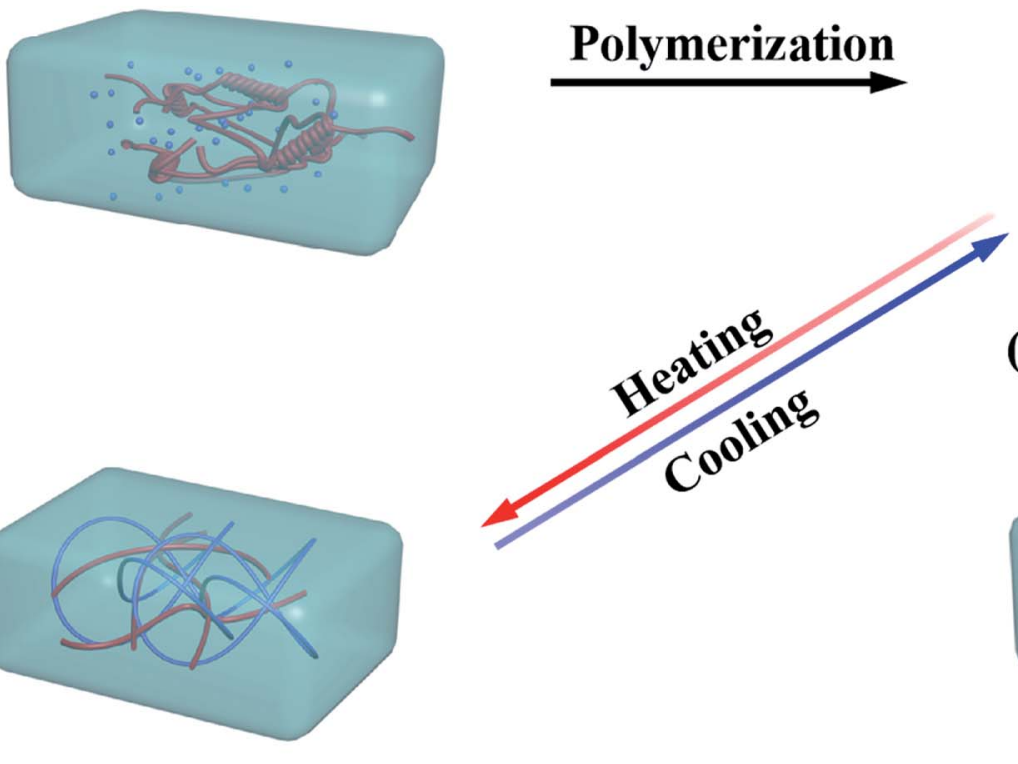

$\underset{\text { Polymerization }}{\longrightarrow}$

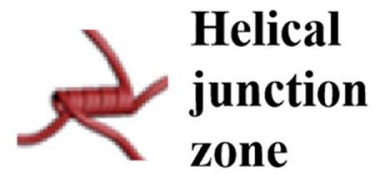

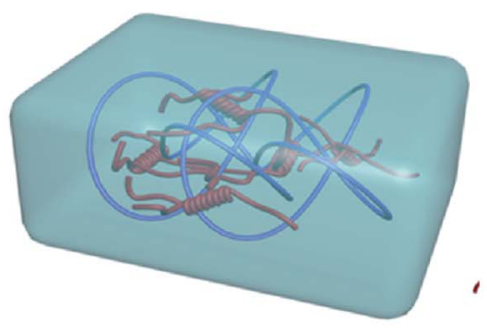

$\left(\mathrm{NH}_{4}\right)_{2} \mathrm{SO}_{4}$

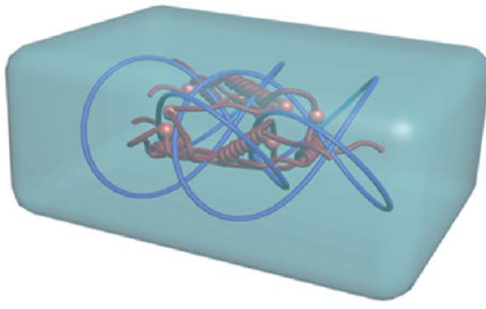

Hydrophobic interaction region

Scheme 1 The mechanisms of the shape memory hydrogel. 


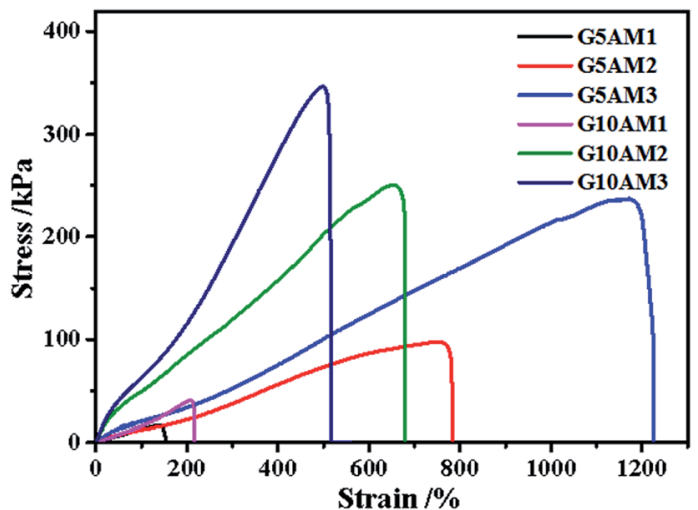

Fig. 2 Tensile stress-strain curves of the gelatin/PAM hydrogels with different gelatin and acrylamide concentrations.

(a)

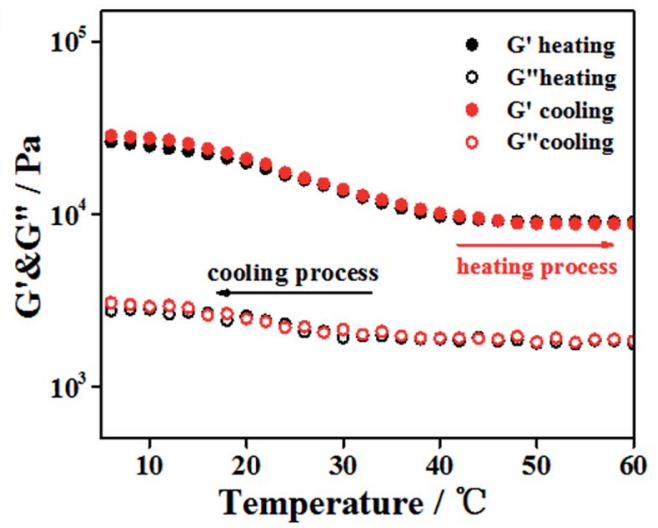

(b)

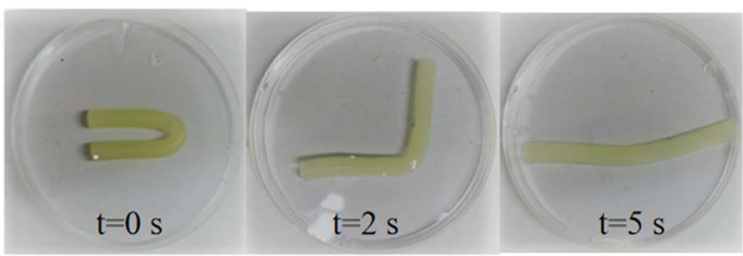

Fig. 3 (a) Dependence of storage modulus $\left(G^{\prime}\right)$ and loss modulus $\left(G^{\prime \prime}\right)$ on temperature. (b) Images demonstrating the transition from the temporary shape to the permanent shape for the thermal-activated SME.

to cool down for the formation of gel at $4{ }^{\circ} \mathrm{C}$. Then it was polymerized under UV irradiation to form an gelatin/PAM DN hydrogel at room temperature for 6 hours. MBA was $0.02 \mathrm{~mol} \%$ relative to the monomers and IRGACURE 2959 was $1 \mathrm{~mol} \%$. In order to investigating the influence of the contents of the gelatin and AM on the mechanical performance of the hydrogel, a series of hydrogels with the different contents of gelatin and AM were prepared. In the experiments, the as-prepared gelatin/PAM hydrogels were designated as GxAMy, in which " $x$ " represents the mass contents of gelatin $(\mathrm{g})$ and " $y$ " represents the molarity of AM (M). Subsequently, the obtained gelatin/PAM hydrogels were soaked in $\left(\mathrm{NH}_{4}\right)_{2} \mathrm{SO}_{4}$ solutions (the concentration of the solutions are $5,10,15,20,25,30,35$, and $40 \mathrm{wt} \%$, respectively) at room temperature for 6 hours to obtain the gelatin/PAM-A hydrogels.

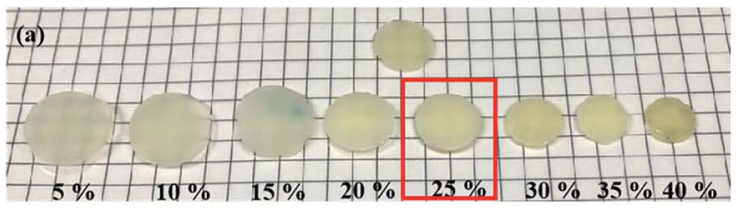

(b)

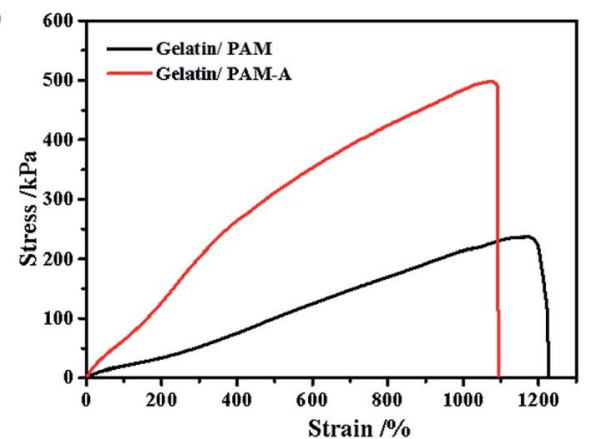

c)

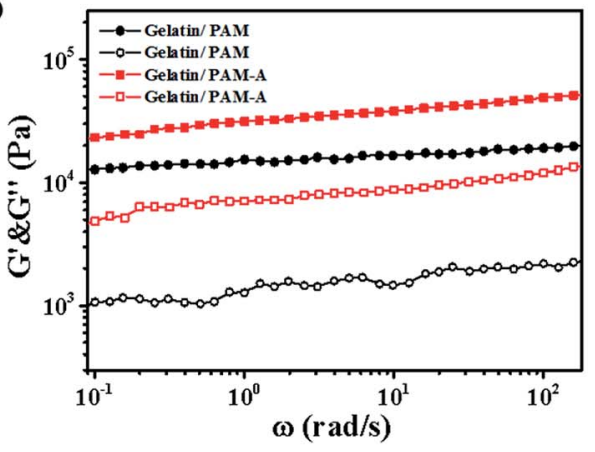

Fig. 4 (a) Photographs of the gelatin/PAM hydrogels after immersion in $\left(\mathrm{NH}_{4}\right)_{2} \mathrm{SO}_{4}$ solutions with various concentrations. (b) Tensile stressstrain curves of the gelatin/PAM and gelatin/PAM-A hydrogels. (c) Frequency sweep data of the gelatin/PAM and gelatin/PAM-A hydrogels in terms of (filled symbols) $G^{\prime}$ and (open symbols) $G^{\prime \prime}$.

\section{Mechanical properties tests}

The mechanical properties of hydrogels were all tested on an electronic universal testing machine (EZTest, SHIMADZU) at room temperature. For the tensile and loading-unloading tests, the hydrogel samples were cut into dumbbell shape with length $13 \mathrm{~mm}$, width $2 \mathrm{~mm}$ and thickness $1.2 \mathrm{~mm}$. In the loading-unloading tests, in order to prevent water evaporating, all the samples were coated with silicone oil. The crosshead velocity was kept at $100 \mathrm{~mm} \mathrm{~min}^{-1}$. For the successive loading-unloading tests, the strain rate was fixed at $150 \mathrm{~mm} \mathrm{~min}^{-1}$. The dissipative energy was calculated from the integral area of the hysteretic circle of the extensionrecovery curve.

\section{Rheological experiments}

The rheological behavior of hydrogels were characterized with a rheometer (TA AR-G2, TA Instruments) equipped with $40 \mathrm{~mm}$ parallel plates. A solvent trap was filled with silicone oil to minimize water evaporation. The storage modulus $\left(G^{\prime}\right)$ and the loss modulus $\left(G^{\prime \prime}\right)$ were measured as a function of angular frequency from 100 to $0.1 \mathrm{rad} \mathrm{s}^{-1}$ at $25{ }^{\circ} \mathrm{C}$. Temperature sweep data were obtained at a fixed frequency $\left(\omega=6.28 \mathrm{rad} \mathrm{s}^{-1}\right)$ and 
(a)
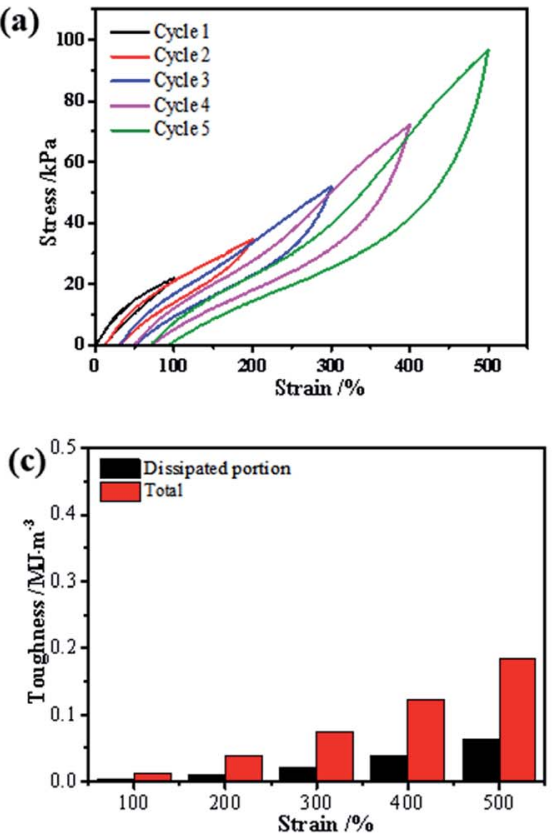

(b)

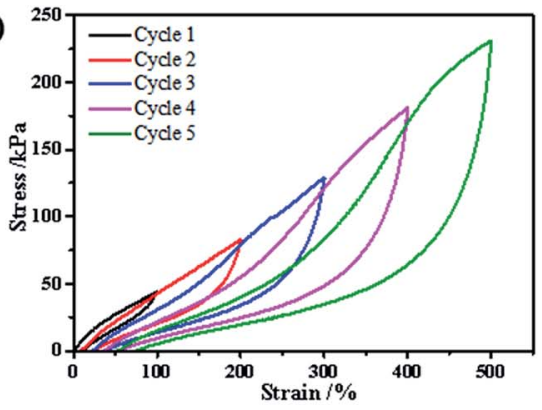

(d)

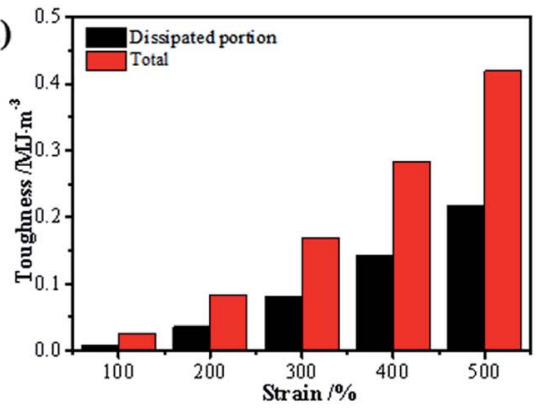

Fig. 5 Loading-unloading tests of the (a) gelatin/PAM hydrogels and (b) gelatin/PAM-A hydrogels with varying maximum tension strains; the calculated total and dissipated toughnesses (curve area) of the (c) gelatin/PAM hydrogels and (d) gelatin/PAM-A hydrogels during the loadingunloading cycles with varying maximum tension strains.

(a) Shape fixity process

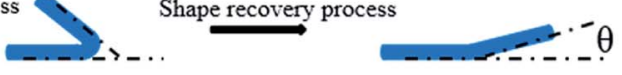

(b)

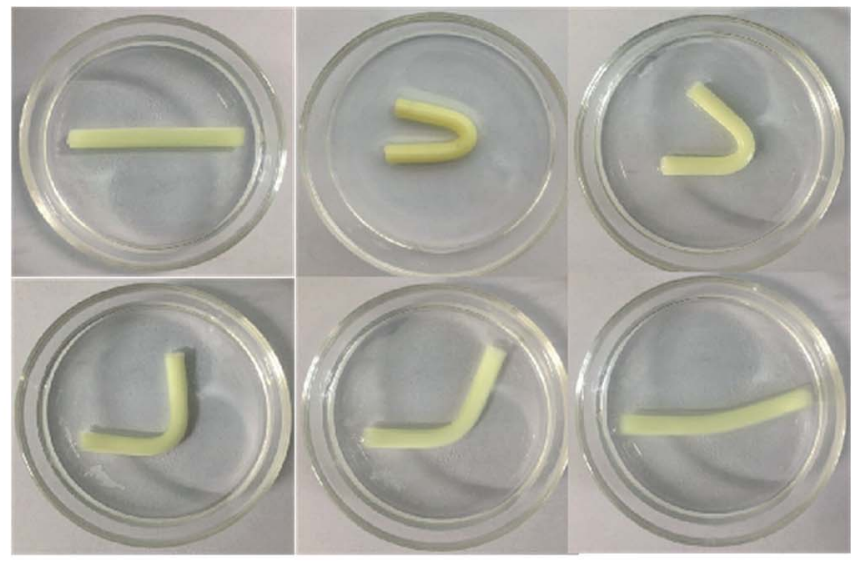

(c)

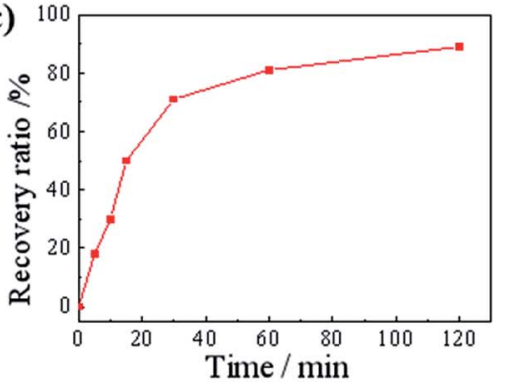

(d)

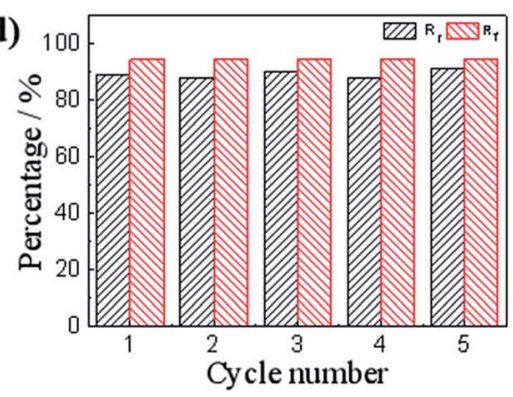

Fig. 6 (a) Schematic illustrations of the shape memory effect. (b) Images demonstrating the transition from the temporary shape to the permanent shape for the salt-activated SME. (c) $R_{\mathrm{r}}$ for the gelatin/PAM hydrogels shown as a function of time at room temperature. (d) Evaluation of the gelatin/PAM hydrogels shape memory cycles at room temperature. 
(a)
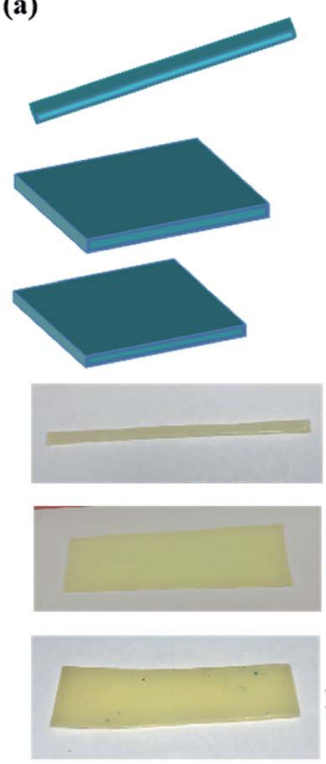

(b)

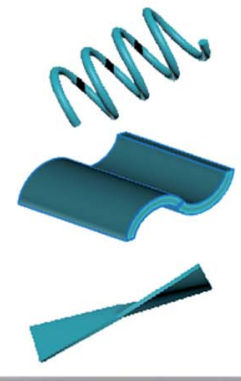

(c)

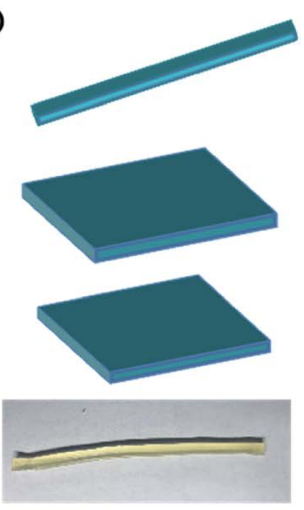

$\underline{\mathrm{K}_{2} \mathrm{CO}_{3}}$

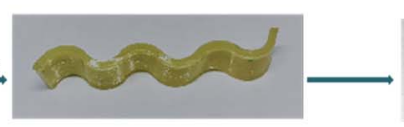

$\underline{\mathrm{K}_{2} \mathrm{HPO}_{4}}$

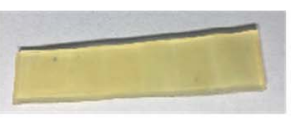

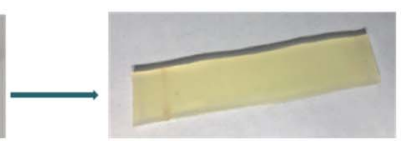

Fig. 7 Photos showing various (a) initial shape, (b) fixed shape and (c) permanent shape at room temperature treated by $\mathrm{Na}_{2} \mathrm{CO}_{3}, \mathrm{~K}_{2} \mathrm{CO}_{3}, \mathrm{~K}_{2} \mathrm{HPO}_{4}$ solution.

strain $(\gamma=0.1 \%)$ covering a range of $5-60-5{ }^{\circ} \mathrm{C}$ (heating and cooling rate: $\left.2^{\circ} \mathrm{C} \min ^{-1}\right)$.

\section{Shape memory evaluation}

The shape memory behaviors of the gelatin/PAM hydrogels were evaluated by bending tests at room temperature. ${ }^{30}$ For the thermal-activated SME, a cylindrical gel sample was immersed in $90^{\circ} \mathrm{C}$ water for $20 \mathrm{~s}$ and then immersed in ice water for $30 \mathrm{~s}$ to fix the temporary shape "U". The shape recover behavior was further observed by immersing hydrogels in $90^{\circ} \mathrm{C}$ water. For the salt-activated SME, a cylindrical gel sample was immersed in $\left(\mathrm{NH}_{4}\right)_{2} \mathrm{SO}_{4}$ solution for $6 \mathrm{~h}$ to fix the temporary shape " $\mathrm{U}$ ". The shape recover behavior was further observed by immersing hydrogels in deionized water. The shape fixity ratio $\left(R_{\mathrm{f}}\right)$ and shape recovery ratio $\left(R_{\mathrm{r}}\right)$ were defined by following equations:

$$
\begin{gathered}
R_{\mathrm{f}}=\frac{\theta_{\mathrm{t}}}{\theta_{\mathrm{i}}} \times 100 \% \\
R_{\mathrm{r}}=\frac{\theta_{\mathrm{i}}-\theta_{\mathrm{f}}}{\theta_{\mathrm{i}}} \times 100 \%
\end{gathered}
$$

where $\theta_{\mathrm{i}}$ is the actually curled angle, $\theta_{\mathrm{t}}$ is the temporarily fixed angle, and $\theta_{\mathrm{f}}$ is the final angle. Each datum for calculating is from three independent measurements.

The shape recovery process was recorded using a digital camera. A series of photographs were picked out to demonstrate the transition from the temporary shape to the permanent shape.

\section{Results and discussion}

The preparation process for gelatin/PAM DN hydrogels was illustrated in Scheme 1. The physically cross-linking triple helices was formed by hydrogen bonds of gelatin at $4{ }^{\circ} \mathrm{C}$. The chemically cross-linked PAM network was formed through the radical polymerization at a total monomer concentration of 1$3 \mathrm{M}$ via UV light. FT-IR analysis was further carried out to verify gelatin/PAM DN hydrogels. As shown in Fig. 1, after polymerization, the absence of monomers was confirmed by the completely disappearance of the peak at $3100-3000 \mathrm{~cm}^{-1}$ corresponding to the carbon-carbon double bond. ${ }^{24}$ The gelatin/ PAM curve has a broadband around $3410 \mathrm{~cm}^{-1}$ and a characteristic peak at $3210 \mathrm{~cm}^{-1}$ due to the stretching vibration of $\mathrm{N}^{-}$ $\mathrm{H}$, which were associated with AM and gelatin. The successful preparation of the gelatin/PAM DN hydrogels was thus confirmed by FTIR spectrum. Upon treatment with $\left(\mathrm{NH}_{4}\right)_{2} \mathrm{SO}_{4}$, the intensity of the amide I bands $\left(1650 \mathrm{~cm}^{-1}\right)$ increased. A peak at $1400 \mathrm{~cm}^{-1}$ for the $\mathrm{C}-\mathrm{H}$ bending vibrations and $\mathrm{CH}_{3}$ symmetrical deformation vibrations became a stronger absorption, which implying the occurrence of stronger hydrophobic interactions..$^{28}$

Then, the mechanical performance of hydrogels with different gelatin and AM concentrations have been tested at room temperature. From the Fig. 2, when the AM concentration was $1 \mathrm{M}$, both of the hydrogels (G5AM1 and G10AM1) exhibited poor mechanical properties. For example, under small tensile stress $(16.21 \mathrm{kPa})$ and strain $(142.99 \%)$, the G5AM1 hydrogel was easily ruptured with a small tensile stress $(16.21 \mathrm{kPa})$ and strain $(142.99 \%)$, as the gelatin contents increased, the maximum tensile stress $(40.83 \mathrm{kPa})$, and strain $(207.92 \%)$ increased little. When the concentration of AM was increased to $2 \mathrm{M}$ or $3 \mathrm{M}$, all of samples show much better mechanical strength. Besides, the mechanical performance also influenced by the gelation concentration. In specifically, when the concentration of AM was set as $2 \mathrm{M}$, the tensile stress at fracture for the hydrogels was increased from 97.22 to $250.56 \mathrm{kPa}$ and 
the failure strain was increased from $656.16 \%$ to $759.78 \%$ as the gelatin content changed from $5 \mathrm{wt} \%$ to $10 \mathrm{wt} \%$. However, the elongation property showed opposite concentration dependent behavior. The fracture stress for G5AM3 was $237.02 \mathrm{kPa}$, while the G10AM3 sample exhibited a fracture stress of $346.08 \mathrm{kPa}$. Although G10AM3 was stronger than G5AM3, the fracture strain of G10AM3 (499.90\%) was much smaller than G5AM3 (1171.15\%). In a word, the G5AM3 hydrogel exhibited excellent mechanical property with tensile stress and strain of 237.02 $\mathrm{kPa}$, and $1171.15 \%$, respectively. Therefore, we chose G5AM3 hydrogel as the model sample and subjected to the following experiments.

It has been mentioned ${ }^{31}$ that gelatin/PAM DN hydrogel can exhibit thermal-activated SME, and we briefly discuss below. Here, triple helices of gelatin were used as the thermal switching segments. Temperature sweep was conducted to investigate the temperature responsive behavior of the gelatin/PAM hydrogels system. Fig. 3a shows the storage modulus $\left(G^{\prime}\right)$ and loss modulus $\left(G^{\prime \prime}\right)$ of gelatin/PAM hydrogels under fixed frequency (6.28 $\mathrm{rad} \mathrm{s}^{-1}$ ) during cooling-heating cycle. The $G^{\prime \prime}$ was lower than the corresponding $G^{\prime}$ in the entire temperature range, suggesting the elastic nature of the gelatin/PAM hydrogels. The $G^{\prime}$ value decreased from 26.2 to $9.1 \mathrm{kPa}$ under the temperature range from 5 to $45{ }^{\circ} \mathrm{C}$, while the $G^{\prime \prime}$ remained fundamentally unchanged in the entire range. When temperature exceeded $45{ }^{\circ} \mathrm{C}, G^{\prime}$ of the gelatin/PAM hydrogel also kept almost a constant in the heating and cooling process. This phenomenon was attributed to the presence of nontemperature responsive chemically crosslinked PAM network, leading to the steady cross-linking density. In essence, the decrease of $G^{\prime}$ with the increasing of temperature contributed to the coil-helix transition of the gelatin in hydrogels. Upon cooling, $G^{\prime}$ and $G^{\prime \prime}$ went back to the original value due to the aggregation of triple helices, indicating thermoreversibility of the hydrogels. Fig. 3b shows the photos of the thermally triggered SME. A gelatin/PAM hydrogel was deformed into U-shape by cooling to $0{ }^{\circ} \mathrm{C}$, and then recovered to a rod-shape within $5 \mathrm{~s}$ after immersing in a water bath at $90{ }^{\circ} \mathrm{C}$. The given angle was $173^{\circ}$, while the final angle was $8^{\circ}$. The thermal-activated SME is attributed to the reversible coil to triple helices transformation of gelatin in response to temperature, and the chemically crosslinked of PAM network function as a fixing phase. Notably, the temporarily fixed shape slightly changed with time even though at freezing temperature (Fig. $\mathrm{S} 1 \dagger$ ).

We then focused on the salt effect on the mechanic behavior of the hydrogel. In order to investigate the effect of ions on the aggregation properties and the performance of gelatin/PAM hydrogels, we treated hydrogels with different ions. Considering that $\mathrm{NH}_{4}{ }^{+}$and $\mathrm{SO}_{4}{ }^{2-}$ are strongly kosmotropic ions that has been examined in the Hofmeister series. ${ }^{28}$ Hence, we chose $\left(\mathrm{NH}_{4}\right)_{2} \mathrm{SO}_{4}$ to act as the switchable agent. Hydrogels were cut into $10 \mathrm{~mm}$ parallel plates and then immersed in $\left(\mathrm{NH}_{4}\right)_{2} \mathrm{SO}_{4}$ solutions at various concentrations $(5,10,15,20,25,30,35$, and $40 \mathrm{wt} \%$ ). The graph in Fig. 5a indicated that hydrogels swelled to some extent at low salt concentration and but shrunk at high salt concentration. Interestingly, sample kept its original volume when the concentration of $\left(\mathrm{NH}_{4}\right)_{2} \mathrm{SO}_{4}$ was $25 \mathrm{wt} \%$. To gain more insight, we conducted tensile and rheological studies of gelatin/PAM and gelatin/PAM-A samples treated by $25 \mathrm{wt} \%$ $\left(\mathrm{NH}_{4}\right)_{2} \mathrm{SO}_{4}$ solution. The tensile stress-strain and frequency data of the hydrogels were presented in Fig. $4 \mathrm{~b}$ and c, respectively. Note that elongation at break of the gelatin/PAM hydrogel was $1171.15 \%$, and the tensile stress was $237.02 \mathrm{kPa}$. After immersing in $\left(\mathrm{NH}_{4}\right)_{2} \mathrm{SO}_{4}$ solution, the tensile stress and elongation at break were $498.41 \mathrm{kPa}$ and $1071.92 \%$, respectively (Fig. 4b). Obviously, the tensile stress of gelatin/PAM-A was apparently higher than the values of the gelatin/PAM hydrogels. Besides, compared to the original sample, elongation of gelatin/ PAM-A hydrogel elongation at break decreased slightly. As expected, after immersing in $\left(\mathrm{NH}_{4}\right)_{2} \mathrm{SO}_{4}$ solution, the reversible physical interaction assisted by Hofmeister effect in the hydrogel generated. As a result, the mechanical properties of the hydrogels were enhanced. The frequency dependence of the $G^{\prime}$ and $G^{\prime \prime}$ of hydrogels at $25{ }^{\circ} \mathrm{C}$ were shown in Fig. $4 \mathrm{c}$. It can be seen that $G^{\prime}$ and $G^{\prime \prime}$ of the gelatin/PAM-A were higher than the corresponding storage $G^{\prime}$ and $G^{\prime \prime}$ of the gelatin/PAM in the entire frequency range (0.1-100 $\left.\mathrm{rad} \mathrm{s}^{-1}\right)$. Gelatin/PAM-A showed an obvious enhancement in terms of $G^{\prime}$ and $G^{\prime \prime}$ compared to gelatin/PAM. Such changing tendency of the $G^{\prime}$ and $G^{\prime \prime}$ was attributed to the formation of the chain bundling and hydrophobic interaction domains which provided the tertiary network within the hydrogels.

In addition, the formation of triple helices and physical interaction was also supported by loading-unloading tests. The loading-unloading cycles of the hydrogel before and after $\left(\mathrm{NH}_{4}\right)_{2} \mathrm{SO}_{4}$ treatment were tested and the stress-strain curves were displayed. Apparently, all of these hydrogels showed a hysteresis loop in the extension-recovery cycles. For gelatin/ PAM, Fig. 5 showed the increase of the dissipated energy from $0.003 \mathrm{MJ} \mathrm{m}^{-3}$ (cycle 1) to $0.06 \mathrm{MJ} \mathrm{m}^{-3}$ (cycle 5). Similarly, it increased from $0.01 \mathrm{MJ} \mathrm{m}^{-3}$ (cycle 1) to $0.22 \mathrm{MJ} \mathrm{m}^{-3}$ (cycle 5) for gelatin/PAM-A. For each cycle, the dissipated energy of gelatin/ PAM-A was higher than that of gelatin/PAM. The hysteresis indicated the energy dissipated during the loading-unloading cycles. ${ }^{32}$ Based on the energy dissipation mechanism, the chemical cross-linking was used to act as a skeleton to maintain structure, while reversible cross-linking served as the sacrificial bonds to dissipate energy. ${ }^{33}$ The aforementioned results suggested that triple helices in gelatin/PAM functioned as the sacrificial bonds; by comparison, not only triple helices but also physical interaction domains assisted by Hofmeister effect in gelatin/PAM-A served as the sacrificial bonds. Consequently, physical interaction domains formed by soaking with $\left(\mathrm{NH}_{4}\right)_{2} \mathrm{SO}_{4}$ solutions could enhance the mechanical properties and apply as temporary cross-links.

After confirming the salt enhanced mechanic property, we then studied the salt-activated $\mathrm{SMH}$ shape memory phenomenon. The shape fixity ratio $\left(R_{\mathrm{f}}\right)$ and shape recovery ratio $\left(R_{\mathrm{r}}\right)$ of the hydrogels were obtained by bending experiments performed on U-shaped samples. Here, the $R_{\mathrm{f}}$ described the efficiency of programming and $R_{\mathrm{r}}$ characterized the ability of the material to memorize the permanent shape. As shown in Fig. 6, a straight strip of gelatin/PAM hydrogel was applied with a continuous external force to bend its shape to a U-shape. Then, it was 
soaked in the $25 \mathrm{wt} \%\left(\mathrm{NH}_{4}\right)_{2} \mathrm{SO}_{4}$ solutions to fix shape with $R_{\mathrm{f}}=$ $94.4 \%$. These physically cross-linking domains assisted by Hofmeister effect could act as temporary cross-links to stabilize the temporary shape. The hydrogel was sequentially placed in deionized water to remove the $\left(\mathrm{NH}_{4}\right)_{2} \mathrm{SO}_{4}$. It was found that the resultant hydrogel recovered to its initial shape within about 120 min and the $R_{\mathrm{r}}$ reached to $89 \%$ (Fig. 6c). As the physical interaction disappeared, the shape gradually recovered to permanent network as a result of extraction of ions with deionized water. In addition, as presented in Fig. S2, $\dagger$ the shape memory behavior of G10AM3 performed the best in fracture stress. G10AM3 sample had a larger modulus and yield stress, which indicated lower fixed ratio $\left(R_{\mathrm{f}}=89.4 \%\right)$. As show in Fig. S2b, $\uparrow R_{\mathrm{r}}$ of G10AM3 hydrogel reached to $85.6 \%$ after $150 \mathrm{~min}$. To examine the repeatability of the shape memory capability, the cycles of shape fixing and recovery were repeated for 5 times (Fig. 6d). The cyclicity was evaluated by measuring $R_{\mathrm{f}}$ and $R_{\mathrm{r}}$ quantitatively for each cycle. ${ }^{34}$ The hydrogel almost remained the same $R_{\mathrm{f}}(\sim 94 \%)$ and could recover to its original shape after five cycles. During each cycle, the $R_{\mathrm{f}}$ and $R_{\mathrm{f}}$ were similar to each other. The reversible physical interaction domains endowed the hydrogel with good reproducible shape memory ability. As a result, the gelatin/PAM hydrogels exhibited excellent shape memory behaviors.

Moreover, considering ions in Hofmeister series would have different effect on aggregation properties, we hypothesized that various kosmotropes ions could also induce SME. The typical order of the anion series is: $\mathrm{CO}_{3}{ }^{2-}>\mathrm{SO}_{4}{ }^{2-}>\mathrm{S}_{2} \mathrm{O}_{3}{ }^{2-}>\mathrm{H}_{2} \mathrm{PO}_{4}{ }^{-}>$ $\mathrm{F}^{-}>\mathrm{CH}_{3} \mathrm{COO}^{-}>\mathrm{Cl}^{-}>\mathrm{Br}^{-}>\mathrm{NO}_{3}{ }^{-}>\mathrm{I}^{-}>\mathrm{ClO}_{4}{ }^{-}>\mathrm{SCN}^{-}{ }^{35}$ The ions on the left are referred as kosmotropes, and those on the right side are called chaotropes. As shown in Fig. S3 and S4, $\dagger$ strong kosmotropic ions induced SME with high fracture stress and $R_{\mathrm{f}}$ while chaotropes ions weaken the fracture stress and couldn't fix temporary shape. Anions were reported to have more pronounced effect than cations. ${ }^{28}$ As show in Fig. S5, $\dagger$ $\mathrm{K}_{2} \mathrm{CO}_{3}$ and $\mathrm{Na}_{2} \mathrm{CO}_{3}$ exhibited similar influence on fracture stress or $R_{\mathrm{f}}(92.2 \%$ and $93.3 \%$, respectively). The recovering process was achieved by immersing hydrogels into deionized water by extracting ions from hydrogels, leading to the higher $R_{\mathrm{f}}$ $\sim 90 \%$ (Fig. S4b and S5b †). The outstanding shape fixed capacities of hydrogels make it possible to deform complicated temporary shapes like spiral and helix. We selected three kinds of salts containing strongly kosmotropes ions for inducing SME. As can be seen from Fig. 7, three straight gelatin/PAM hydrogels were deformed to spiral, wavy and helix shapes. The resulted shapes were fixed by soaking with $\mathrm{Na}_{2} \mathrm{CO}_{3}, \mathrm{~K}_{2} \mathrm{CO}_{3}$, and $\mathrm{K}_{2} \mathrm{HPO}_{4}$ solutions, respectively. When the hydrogels immersed into deionized water, the temporary shapes could recover to their original shapes. These results suggested that gelatin/PAM hydrogels can be easily fixed to any desirable form by immersing into saline solution, which would extend extending the great potential application range.

\section{Conclusions}

In summary, a type of gelatin/PAM DN hydrogel was successfully synthesized through polymerization of AM in the presence of gelatin. We demonstrate the thermal and salt-activated shape memory effects in such biopolymer based hydrogels for the first time. The PAM network serves as a permanent network. The gelatin not only endows gelatin/PAM DN hydrogel with temperature-responsiveness but also brings the hydrogel with salt-responsiveness. Thus, we believe that the facile strategy could provide new opportunities with regard to the practical application. In addition, the presented strategy could broaden the list of SMHs and apply to other collagen systems.

\section{Conflicts of interest}

There are no conflicts to declare.

\section{Acknowledgements}

This work was supported by the National Science and Technology Major Project of the Ministry of Science and Technology of China (2016ZX05016), the National Science and Technology Major Project of the Ministry of Science and Technology of China (2016ZX05046), and the National Natural Science Foundation of China (Grant no. 51273189).

\section{Notes and references}

1 T. R. Hoare and D. S. Kohane, Polymer, 2008, 49, 1993-2007.

2 X. Le, W. Lu, J. Zhang and T. Chen, Adv. Sci., 2019, 6, 1801584.

3 N. Annabi, A. Tamayol, J. A. Uquillas, M. Akbari, L. E. Bertassoni, C. Cha, G. Camci-Unal, M. R. Dokmeci, N. A. Peppas and A. Khademhosseini, Adv. Mater., 2014, 26, 85-124.

4 J. Hao and R. A. Weiss, ACS Macro Lett., 2013, 2, 86-89.

5 W. Guo, C. H. Lu, R. Orbach, F. Wang, X. J. Qi, A. Cecconello, D. Seliktar and I. Willner, Adv. Mater., 2015, 27, 73-78.

6 W. Feng, W. Zhou, S. Zhang, Y. Fan, A. Yasin and H. Yang, RSC Adv., 2015, 5, 81784-81789.

7 T. Zhao, M. Tan, Y. Cui, C. Deng, H. Huang and M. Guo, Polym. Chem., 2014, 5, 4965.

8 G. Li, Q. Yan, H. Xia and Y. Zhao, ACS Appl. Mater. Interfaces, 2015, 7, 12067-12073.

9 Y. N. Chen, L. Peng, T. Liu, Y. Wang, S. Shi and H. Wang, ACS Appl. Mater. Interfaces, 2016, 8, 27199-27206.

10 C. Lowenberg, M. Balk, C. Wischke, M. Behl and A. Lendlein, Acc. Chem. Res., 2017, 50, 723-732.

11 H. Meng, J. Zheng, X. Wen, Z. Cai, J. Zhang and T. Chen, Macromol. Rapid Commun., 2015, 36, 533-537.

12 C. Bilici and O. Okay, Macromolecules, 2013, 46, 3125-3131.

13 N. Li, G. Chen, W. Chen, J. Huang, J. Tian, X. Wan, M. He and H. Zhang, Carbohydr. Polym., 2017, 178, 159-165.

14 B. Xu, Y. Zhang and W. Liu, Macromol. Rapid Commun., 2015, 36, 1585-1591.

15 P. J. Skrzeszewska, L. N. Jong, F. A. de Wolf, M. A. Cohen Stuart and J. van der Gucht, Biomacromolecules, 2011, 12, 2285-2292.

16 K. Miyamae, M. Nakahata, Y. Takashima and A. Harada, Angew. Chem., Int. Ed., 2015, 54, 8984-8987. 
17 W. Lu, X. Le, J. Zhang, Y. Huang and T. Chen, Chem. Soc. Rev., 2017, 46, 1284-1294.

18 B. Gyarmati, B. Á. Szilágyi and A. Szilágyi, Eur. Polym. J., 2017, 93, 642-669.

19 C. Bilici, V. Can, U. Nöchel, M. Behl, A. Lendlein and O. Okay, Macromolecules, 2016, 49, 7442-7449.

20 Z. X. Zhang, K. L. Liu and J. Li, Angew. Chem., Int. Ed., 2013, 52, 6180-6184.

21 X. Li, S. Xu, Y. Wang, Z.-Y. Hu and R. Wang, Colloid Polym. Sci., 2019, 297, 503-512.

22 H. Xiao, C. Ma, X. Le, L. Wang, W. Lu, P. Theato, T. Hu, J. Zhang and T. Chen, Polymers, 2017, 9, 138.

23 X. Le, W. Lu, J. Zheng, D. Tong, N. Zhao, C. Ma, H. Xiao, J. Zhang, Y. Huang and T. Chen, Chem. Sci., 2016, 7, 67156720.

24 K. Peng, K. Yang, Y. Fan, A. Yasin, X. Hao and H. Yang, Macromol. Chem. Phys., 2017, 218, 1700170.

25 L. J. Del Valle, A. Diaz and J. Puiggali, Gels, 2017, 3, 27.

26 K. Ullah, S. Ali Khan, G. Murtaza, M. Sohail, Azizullah, A. Manan and A. Afzal, Int. J. Pharm., 2019, 556, 236-245.
27 J. Huang, L. Zhao, T. Wang, W. Sun and Z. Tong, ACS Appl. Mater. Interfaces, 2016, 8, 12384-12392.

28 Q. He, Y. Huang and S. Wang, Adv. Funct. Mater., 2018, 28, 1705069.

29 X. Chen, S. C. Flores, S. M. Lim, Y. Zhang, T. Yang, J. Kherb and P. S. Cremer, Langmuir, 2010, 26, 16447-16454.

30 W. Nan, W. Wang, H. Gao and W. Liu, Soft Matter, 2013, 9, 132-137.

31 N. Li, W. Chen, G. Chen and J. Tian, Carbohydr. Polym., 2017, 171, 77-84.

32 Z. Wang, W. Li, X. Yang, J. Cao, Y. Tu, R. Wu and W. Wang, Mater. Today Commun., 2018, 17, 246-251.

33 T. Nakajima, H. Sato, Y. Zhao, S. Kawahara, T. Kurokawa, K. Sugahara and J. P. Gong, Adv. Funct. Mater., 2012, 22, 4426-4432.

34 Y. Fan, W. Zhou, A. Yasin, H. Li and H. Yang, Soft Matter, 2015, 11, 4218-4225.

35 Y. Zhang, S. Furyk, D. E. Bergbreiter and P. S. Cremer, J. Am. Chem. Soc., 2005, 127, 14505-14510. 